\title{
A STUDY OF THE EFFECT OF RIGHT FRONTAL LOBECTOMY ON INTELLIGENCE AND TEM- PERAMENT
}

BY

\section{T. LIDZ}

From the Research Unit, National Hospital, Queen Square, London

(RECEIVEd 23Rd February, 1939)

A VARIETY of psychological examinations and related observations were made upon a patient before and after removal of the right prefrontal regions for an oligodendroglioma. Although lesions of the prefrontal regions have long been associated with disturbances of intellect and personality, few attempts have been made to measure changes subsequent to right frontal lobectomy, though two cases have been studied thoroughly following bilateral frontal lobectomy (Brickner, 1932, and 1936; Ackerly, 1935). The present case afforded excellent opportunity for study because of the minimal symptomatology prior to operation and because of the patient's high intelligence quotient.

\section{Case Report}

Mr. C. J., aged 37, entered the National Hospital with the complaint of two attacks of unconsciousness during the four months preceding admission. He had suffered from vague headaches for several years which resembled more closely a pressure on the scalp than a true pain. They were not confined to a single location, but shifted about. Straining and change of posture did not affect the headaches, which were never more than mildly annoying, never interfered with sleep, and usually disappeared after a good night's rest. The patient believed that the headaches were caused by the annoyances in his position as a clerk in a cloth firm, which he had held for many years and which offered little prospect for advancement. Shortly before admission he changed his occupation and the headaches diminished in frequency and severity. Upon direct questioning C. J. recalled that for two years there had been episodes of momentary blankness ; a sensation which passed away before he was able to focus his attention upon it. In February 1938 he suffered an attack of unconsciousness. While engaged in conversation he felt his eyes and mouth twitching, experienced a sensation of vagueness, and after a few seconds fell to the floor and was unconscious for five or ten minutes. After regaining consciousness he vomited. There was no incontinence or tongue biting. For several days after the attack he felt weary and was not disposed to work. Six weeks later he suffered a second, less severe attack. Following a set of tennis which he had played with normal skill and facility, the same twitching of the face occurred. His wife noted that it was on the left side. After the "queer" sensation recurred, he fell and was unconscious for a few minutes. There were no movements apart from the twitching of the left side of the face. During April 
and May he again had the peculiar feeling accompanied by the sensation of twitching of the left side of the face without any actual twitching. Six such attacks took place, during which people noted that he had a dazed appearance for a few seconds, and the patient said that he felt as if he had been staring but could not stop.

Family history was irrelevant. Personal history revealed normal development. He had the usual elementary school education and had been considered a superior student. He had worked as a clerk in a cloth firm for many years until shortly before admission. The marriage of six years' duration was happy. The patient was continent in his habits and industrious at work.

Physical examination on 2nd June, 1938, and upon repeated occasions prior to the operation on 10th July revealed no abnormalities. The patient was right-handed. Fundi and visual fields were normal and there was no anosmia. X-ray of the skull showed a small right frontal calcification suggestive of neoplasm. Lumbar puncture revealed no increase in pressure, no cells, and a total protein of $45 \mathrm{mgm}$. per cent.

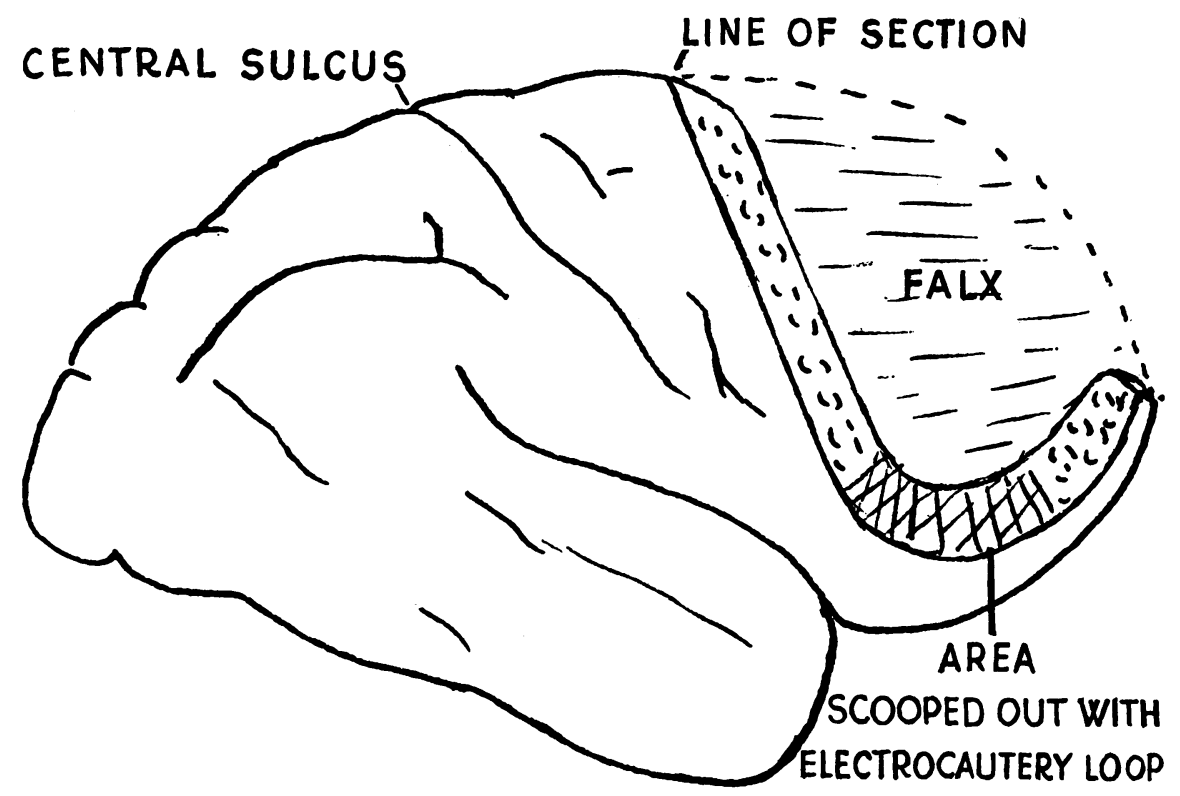

Fig. 1.-Schematic representation of operative procedure.

The patient was placed on a small daily dosage of phenobarbital and there were no attacks during the time he was subjected to the preliminary psychological tests.

Operation.-Mr. Geoffery Jefferson performed a right frontal lobectomy on July 10, 1938. A well-defined tumour was visible on the cortex of the prefrontal area, but there was sufficient normal cortex separating the tumour from the pre-central area to permit lobectomy through normal tissue without endangering the motor cortex. The lobectomy was incomplete anteriorly, where a small area of cortex was left intact at the very pole because of a leash of vessels between the cortex and the dura. After the major portion of the lobe had been removed, a considerable amount of the base was scooped out with a cautery loop, for it appeared as if the tumour were infiltrating the basal region. The anterior horn of the ventricle was not involved in the tumour or the removal of the lobe. The plan of dissection is shown in Fig. 1. It was believed that the lobectomy was made through the anterior portion of Area 6 of Brodman. 
Although the base of the lobe was incompletely removed, there is little doubt that the removal and cauterization completely destroyed the functioning of this region.

The physical status following operation was but slightly altered. The power of the left arm and leg was slightly diminished. The deep reflexes on the left side were slightly more brisk than on the right, at first in both upper and lower extremities, but after a month only in the leg. The plantar response remained flexor ; a grasp reflex was not present and equilibratory disturbances were not elicited. There was no anosmia.

\section{Psychological Observations}

To permit comparative graded testing before and after the operation an attempt was made to base the experimental data, as far as possible, on tests which were paired and numerically measurable. A battery of tests was selected of sufficient breadth to uncover defects or alterations in mentation even if not anticipated from previous writings. As it seemed possible that modifications of behaviour might occur which were not amenable to testing, the observations included estimations of the patient's behaviour by persons who were in close contact with him. He was resident in the hospital for forty days before and over forty days following the operation.

Neither C. J. nor his wife had noted any change in his behaviour during the months or the few years preceding hospitalization. They were questioned at first in general fashion, and then with specific reference to the symptoms noted by Brickner (1936) in his patient who had both frontal lobes removed. Any change in character with reference to ambition, drive, judgment, retentive capacities, evasiveness, initiative, euphoria, compulsiveness, boasting, appreciation of the situation, were denied. There had been no diminution in his ability to carry out business or domestic duties aside from the period of fatigue following the convulsions.

The patient's behaviour before and after operation was noted by the physicians, nurses, fellow patients, his wife, and himself. For three or four days after the operation under general anæsthesia, the patient felt that he could not be bothered to do anything and it was necessary to urge him to eat. The feeling gradually disappeared and was scarcely noticeable after two weeks, though not until a month had elapsed did he feel that his normal drive had returned completely. For two or three days he felt compelled to count things, counting window-panes, beds, etc., but on the fourth day the compulsion had disappeared. For the same length of time there was a difficulty in initiating speech. Appreciation of spoken and written language was not impaired, nor was there hesitancy in knowing what he wished to say, but solely in the initiation of the action of speech, and once started words flowed normally. Alertness was unimpaired. Immediately after awakening from the anæsthesia, the patient directed the nurse to give his wife a letter written on the preceding day, recalling where he had placed it. There was no difficulty in comprehension of remarks and witticisms.

A month after the lobectomy C. J. played whist and snooker as well as ever, according to his own critical judgment and that of patients who had been on the ward with him prior to the operation. He read the newspapers intelligently 
and displayed concern over the international situation. He was anxious to return to work and feared that weight loss might hinder a rapid return to his duties as a salesman. Interest was taken in the examinations and he was hopeful that his mental faculties had not been impaired. Neither the patient nor his wife could note anything in his general make-up and habits which had been altered. He was careful to shave before visits from his wife and on one occasion requested that the tests be shortened to allow him opportunity to tidy up before visitors arrived.

The writer, who did most of the testing, observed no alteration in the patient's desire to co-operate or co-operation, in his demeanour or attitude, although many of the tests were arduous and annoying, demanding strict attention and ability to persevere.

\section{Intelligence Tests}

Several general intelligence tests were utilized which varied in the approach to the measurement of intelligence.

The revised Stanford Binet Test (Terman and Merrill, 1937). Forms L and $\mathbf{M}$ were used as a well standardized method of testing adult intelligence. The results pre- and post-operatively were identical both as to score and scatter.

Pre-operative : June 28. Form L. Mental Age 20 years 11 months. I.Q. 139 Post-operative : Aug. 17. Form M. Mental Age 20 years 11 months. I.Q. 139

The Cattell Test, Scale III, Forms A and B (Cattell, 1935). The scale was designed for testing mental ages of fifteen years and upwards, with emphasis placed upon the " $\mathrm{g}$ " factor of intelligence. The examinee works alone, answering by underlining, checking, and filling in. The test is divided into several sections and although results before and after operation were within the limits expected in retesting, it may be worthy of note that a fall in performance occurred in the difficult "inferences" which was partially compensated by improved performance in other sections.

Pre-operative : July 4. Form A. I.Q. 143.

Post-operative : Aug. 4. Form B. I.Q. 137.

The McCall Intelligence Test, Form 1 (McCall and Herring, 1937), though primarily intended for children, seemed to offer a test which called for flexibility and might afford a means of examining the patient's ability to "readjust his mental set by seeking to divine thirty or more principles of relationship among ideas. That such flexibility is called for is not often noticed when the test is first seen, because the items look so much alike" (McCall and Herring, 1937). Examination of the ability to readjust rapidly from one mode of grouping to another was desired because of the degradation of such flexibility in aphasias, and, according to Goldstein (1937), in frontal lesions. Although pre- and postoperative results did not vary, the I.Q. as measured by this test was much lower than on the other tests, and, as frontal lobe function may have been disturbed before lobectomy, may be significant. However, judgment must be withheld 
until the test has been given a more thorough trial in adult testing. As the test had not been paired at the time of the examinations, the same form was necessarily repeated, but the large number of items and the nature of the test must almost eliminate improvement from one time repetition.

Pre-operative : Form 1-Correct answers, 102. I.Q. 95.

Post-operative : Form 1-Correct answers, 107. I.Q. 99.

The Kohs Block Design Test (Kohs, 1923) involves the copying of seventeen designs of graded difficulty with cubes differently coloured on each surface. It is a non-verbal test measuring through a seventeen-year-mental age level which requires clarity of figure conception, rapidity of synthesis, and manual dexterity. Scoring is based upon the amount of time and number of moves required to form each pattern. A perfect score was attained on both occasions and therefore the test could not be used as a measure of intelligence, but the facility required to attain the maximal score lent importance to this test.

Pre-operative : June 28. Maximal Score 131 points.

Post-operative : Aug. 17. Maximal Score 131 points.

Porteus Maze Tests (Porteus 1924) : a performance test which is said to correlate better with social and industrial capacity than the Binet Test. It offered a test of visual analysis and the capacity to grasp the problem as an entity. The test proved too simple, both before and after lobectomy, as all of the mazes through the fourteen-year level were solved with ease.

In an effort to obtain a wide variety of tests which might uncover defects which had not been anticipated, a group of tests unsuited for quantitative measurement was used. L. L. Thurstone in his monograph Primary Mental Abilities (Thurstone, 1938) gives samples from a battery of fifty-six tests which had been used in his analysis of intelligence into factors. Approximately forty of the test samples were given the patient both before and after the operation. The samples given were not full enough and frequently not difficult enough to detect minor differences in capacity subsequent to the operation, but helped to assure that little which is capable of being tested in the sphere of intelligence was completely overlooked. The tests in the effort to examine single factors of intelligence are frequently highly abstract. As no variations in the ability of the patient were found, the tests will not be described. Among the factors sampled were : Abstraction of words and symbols; verbal associations ; numerical, verbal and spatial reasoning ; space and form perception ; rhythm; right and left recognition; sound perception. Had alterations occurred, they would have been analyzed more readily than from changes in the general intelligence tests.

Graded mental arithmetic problems from Burt's Handbook of Tests (Burt, 1933) showed no appreciable change in performance. The patient worked more carefully and slowly post-operatively and showed as good or better grasp of the problems.

Pre-operative : Fourteen year level-6 of 9 correct.

Post-operative : Fourteen year level-7 of 9 correct. 
Graded reasoning problems from Burt's Handbook of Tests gave identical performance on both occasions, when the same single problem was missed.

\section{Memory Tests}

Word memory : a list of 25 words was given the patient to study for two minutes. After an interval of a minute he was asked to select the 25 words from a list of 75, constructed by adding two words to each of the words on the original list and which were associated by sound, spelling, association, or by addition or subtraction of prefixes and suffixes to the original words. Effort was taken to make the two tests of equal difficulty. The patient was unable to equal his amazing pre-operative score, but the slight post-operative deterioration was of doubtful significance, as it was still a better performance than any obtained from control subjects of high intelligence.

Pre-operative : June 22, 21 of 25 words correct.

Post-operative : Aug. 9, 18 of 25 words correct.

Comparative tests in the ability to memorize were made and utilized to test the patient's ability to retain newly gained knowledge through the operation. A week before operation he was given four lines of Latin poetry, 22 words in length, to memorize. As he was ignorant of foreign languages, it was equivalent to memorizing nonsense words. The verse was learned over night without great difficulty. Eighteen days after the operation he was able to recall but two lines, but after thinking about them for a day or two he reconstructed the four lines without error. On 3rd August he was given four lines of German poetry, also nonsense to him. There was not the same readiness to undertake the task, and for a few days he did not try to learn them, but when asked a second time, he memorized the 24 words over night and judged that the task had been no more difficult for him than before operation. The lines were retained for at least several weeks.

Four lines of light English verse were memorized after three or four hearings both before and after lobectomy, and the ditty learned before the operation was recalled intact after it.

Several lines 43 words in length concerning muscle physiology were memorized by the patient with relatively little difficulty a week before operation. The complexity of the meaning made it difficult for the patient, untrained in science, to comprehend the meaning without a goodly amount of reasoning. It had been anticipated that the words would be memorized as English nonsense, but the patient was able to give a fairly good account of the meaning and had memorized by puzzling it out. Twenty-five days after the operation the lines were recited without hesitation or error. An equivalent passage of 41 words concerning nerve physiology and even more difficult to comprehend was learned in the same manner on 3rd August. Again the lines were retained for at least a few weeks. 


\section{Miscellaneous Tests}

To obtain a general impression of the patient's grasp, alertness, orientation, and ability to synthesize he was requested to write a ten-minute essay. Before operation he was asked to write about the complicated foreign situation, and following upon the Russo-Japanese conflict in Siberia which had arisen rather suddenly since his operation. Both themes displayed a fine grasp of the situation. In the first essay he utilized 220 words and mentioned in natural sequence : the Versailles Treaty as the source of the ensuing difficulties ; Hitler's rise to power ; Germany's desire to regain lost territory ; the unpreparedness of England ; the annexation of Austria ; the Abyssinian conquest and the war in Spain as causes of England's haste to rearm ; the steadying influence of England's power ; the importance of the friendship between England and France ; the poor financial situation of France. In the second essay, written on 9th August, it was seen that the patient had not lost his interest in current affairs or his ability to comprehend them and to organize them in his own mind. In 205 words he mentioned : Japan's fear of Russian intervention in the war with China ; the threat of the Vladivostok air-base to Japan ; Russian preparations in Siberia ; the independence of the Siberian army as to military and food supplies; the quality of the Russian armaments; the fear that Germany might invade the Ukraine if Russia became involved in the Far East ; the importance of the Ukraine to Germany. In some respects the essays appear to give the most concrete evidence of the preservation of the integrity of the patient's mental processes.

Test of following directions. The ability to carry out a series of simple commands in rapid sequence was tested. Thirty-five commands were given as rapidly as the patient could carry them out and were performed without error both before and after the operation. Rapidity of comprehension and the ability to shift from one type of performance to another were tested in this manner.

\section{Will Temperament Test}

A mode of testing for those characteristics which may, in a general way, be termed alterations in temperament or personality, so frequently significant in frontal lesions, was desired. The Downey Will Temperament Test (Downey, 1923) seemed suited for the testing of such factors as ability to persevere and concentrate, loss of restraint, compulsiveness, decisiveness, etc. The test utilizes, for the most part, motor function in the form of handwriting. The person examined writes his name and "The United States of America" under specific conditions : at normal tempo, speeded, with maximal slowness, disguised, copying samples, with distraction, against resistance, and on limited space. Speed of decision and reaction to contradiction are measured by techniques other than writing. Motor impulsion and inhibition may be pivotal points in the test. The tests are divided to measure twelve factors : speed of movement : freedom from load ; flexibility ; speed of decision ; motor impulsion; reaction to contradiction; resistance to opposition ; finality of 
Name C.J. JULY 5, 1938

Speed of Movement VI-1

Freedom from Load II-1, 2; VI-1, 2

Flexibility VIII

Speed of Decision I

Motor Impulsion X

Reaction to Contradiction XI

Resistance to Opposition XII

Finality of Judgment XIII

Motor Inhibition VII

Interest in Detail LX

Coördination of Impulses V

Volitional Perseveration VIII-2

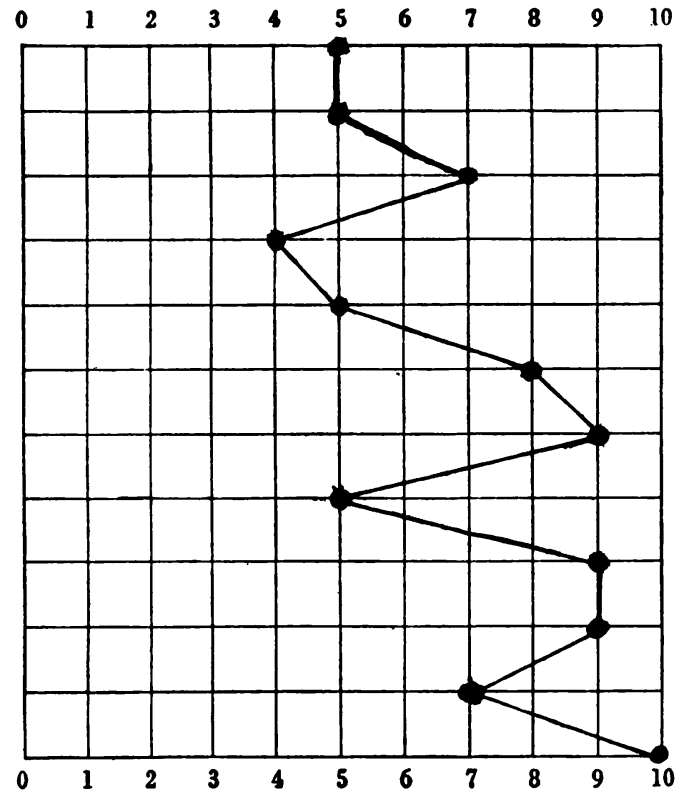

Name C.J. AUGUST 6, 1938

Freedom from Load II-1, 2; VI-1, 2

Flexibility VIII

Speed of Decision I

Motor Impulsion X

Reaction to Contradiction XI

Resistance to Opposition XII

Finality of Judgment XIII

Motor Inhibition VII

Interest in Detail IX

Coördination of Impulses V

Volitional Perseveration VIII-2

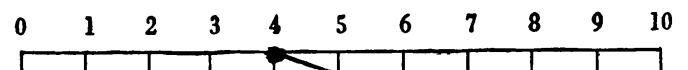

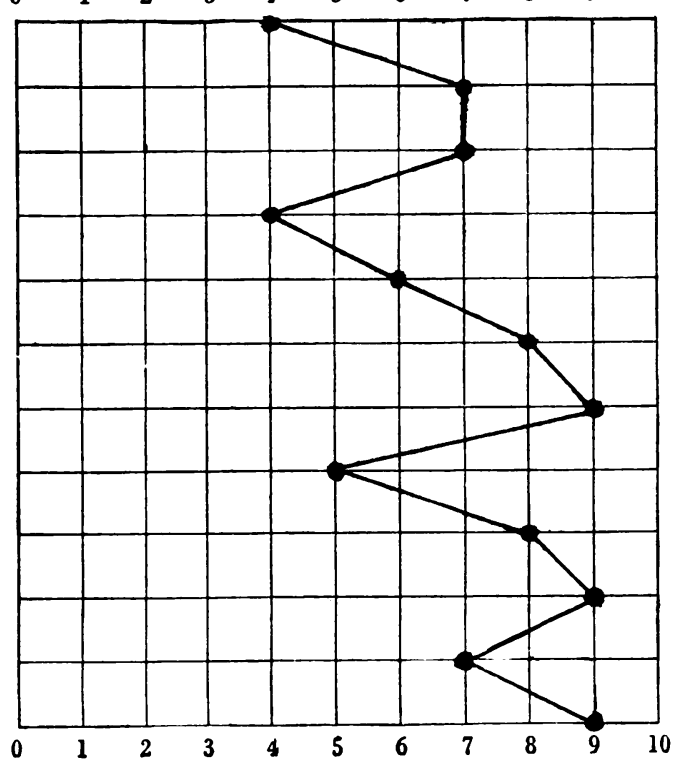

TABLE I.-Graphic Representation of Results of Downey Will Temperament Tests 
judgment ; motor inhibition ; interest in detail ; co-ordination of impulses ; and volitional perseveration. Whether or not the test measures the traits listed is not a concern of this paper. It is believed that the test measures something or other concerning the temperament and that the nature of the test is such that one would anticipate a modification in the responses to some of the tests if the personality were altered to any noticeable extent.

The results of the test may be listed in the form of a graph called the Will Profile. The results of each division of the examination are scored from one to ten according to a set of standards statistically determined by the originator. The patient was subjected to the test on 5th July, prior to lobectomy, and it was repeated on 6th August, following the lobectomy. The results are given in Chart 1. The similarity of the graphs is striking, and is more so if contrasted with graphs of other persons. The scores differ by more than one point on but one item, Freedom from load. While this trait is important in the consideration of frontal lobe function, the change in actual performance was too slight to permit any significance to be attached to the change in this isolated case. It is to be emphasized that the test was not given to gain an estimate of the patient's personality, the original purpose of the test, but as a means of uncovering any modification which might have occurred.

\section{Discussion}

The results of the battery of tests described indicate that the lobectomy produced no modification of intellectual or personality functions. The differences in performance before and after operation were negligible; the few improvements and deteriorations balanced. It is possible that a few items might be of significance ; the difference of two points on the test of Freedom from load, and the failure of the I.Q. as measured by the McCall Test to reach the high level shown in all other tests. If such changes were recurrent in a series of cases some importance might be placed upon them, but it does not seem wise to attempt to evaluate these minor changes in the present case.

The case offered an opportunity for pre- and post-operative testing in a case not confused by preoperative symptomatology or by increase in intracranial pressure. The criticism that the tumour had already destroyed the functioning of the areas removed is justified. Fortunately the patient's high I.Q. almost eliminates the possibility that the tumour had decreased his intelligence, while behaviour and performance in other spheres were so normal or of such high calibre that any change which might have occurred must have been slight. It is possible that the slow-growing tumour had completely destroyed the functioning of the right prefrontal areas and the functions had been completely taken over by the left side. If this was the case it is still interesting that destruction of the entire prefrontal regions on the right can occur without producing demonstrable defects. Though the presence of marked pathology in the areas removed prevents the consideration of the case as a definitive one in settling the problems of functions of the right prefrontal regions, it is believed that it more closely approximates the ideal than any previously reported. 
A survey of the literature reveals slight differences of opinion concerning the effects of right frontal lobectomy. The number of observations concerning intellectual functions after lobectomy are few. Two cases were reported by Penfield and Evans (1932 and 1935). One case in which the frontal pole alone was removed showed no alterations. The other case, even though no direct tests were performed, is most important as the patient was related to one of the observers and accurate judgment of behaviour was possible. The patient considered herself to be a "little slow," and the authors summed up the defect as " a lack of capacity for planned administration." German and Fox (1932) also reported two cases of right frontal lobectomy in which intellectual functions were tested. Unfortunately both of the patients were left-handed; one showed aphasic symptoms and clearly comes under the study of left prefrontal function; in the second cerebral dominance was not clear, as the patient suffered no aphasia though definitely left-handed. The resultant disturbances in the second case were slight; a slight euphoria and difficulties in spatial localization. Disturbances in spatial localization were not found in the present case as tested by several samples in the Thurstone battery.

Jefferson (1937) reported a series of eight frontal lobectomies in which the patients were observed for intellectual and emotional deficits following operation. There were five cases of right frontal lobectomy, three of whom were living and well when the article was written. It could be stated definitely that in two of the three cases there were no abnormalities which could be noted by the surgeon, patient, or family, and while the third case showed a mild memory defect, the operation had been too recently performed to judge whether or not the loss would be permanent. The three cases of left frontal excision likewise showed no significant changes, but comment was made that one patient was slightly lacking in reserve, another remained slightly facetious, and the third, who suffered a transient post-operative aphasia, appeared a trifle slow and diffident. As all three left frontal cases suffered from fairly severe symptoms pre-operatively, it is difficult to judge whether the residua were caused by the removals. Jefferson does not believe that there was any difference between the results of the right and left-sided operations, and considers that bilateral damage is necessary for the production of symptomatology. Psychometric tests were not made and the author warns that none of the patients were of " any considerable mental stature ; their occupations such as would easily bring to light faults in the higher synthesis of ideas" (Jefferson, 1937). The present case confirms the conclusions concerning the absence of mental deterioration following right frontal lobectomy, and on the basis of the detailed studies in a person of high intelligence can further emphasize that mentation can remain intact.

As the series of tests was originally undertaken to ascertain whether or not a defect in mentation could be found in this patient which might be of diagnostic aid in future cases with absence of physical signs, the writings of Goldstein (1936 and 1937) are of particular interest. Goldstein believes that a patient with an extensive frontal lobe lesion, though more so if it is left-sided, will show defects in abstract thinking and performance as contrasted with the concrete. He states, "If the examination is undertaken in the proper way and if the 
damage is not too slight, the characteristic modification will, without doubt, be discoverable in some examples of performance" (Goldstein, 1936). A review of the battery of examinations utilized will reveal a large number of tests of abstract performance. The ability to solve complex verbal and symbol codes in the Thurstone and Cattell tests; the performance with syllogisms, pattern analogies, block counting, surface development tests, and figure classification tests from the Thurstone samples are a few specific examples of abstract performance. The ability to play whist, the comprehension of many of the problems given, and the very high I.Q. rating would scarcely be possible if abstract thinking had suffered markedly. Memorizing passages on muscle and nerve physiology, subjects totally foreign to the patient, with the aid of even general comprehension of the meanings, would not be anticipated from Goldstein's papers. It is true that on difficult " inferences" and several other very abstract tests the patient did not attain as high a level as on some other tests, but the failure was never pronounced, always of questionable significance in a person untrained to such material, and never of diagnostic significance. It seems fair to say that in the present case at least obliteration of the right prefrontal areas produced no loss in the realm of abstract performance, at least not an alteration which might be of diagnostic significance.

\section{Summary}

Intellectual functions and personality traits were studied in a man of high intelligence before and after the removal of his right prefrontal region because of an oligodendroglioma which had produced no signs and but mild symptoms. The section was performed bordering upon or through the anterior portion of Area 6 of Brodman. No physical symptoms ensued aside from a slight increase in the deep reflexes of the left extremities. A battery of tests selected to test intelligence collectively and according to factors and to attempt to test for alterations in temperament, and careful observation of the patient's behaviour, revealed no changes. Deterioration in ability for abstract performance was not found. The results of the tests coincided with the impression of both the patient and the several persons in position to observe him carefully.

Miss C. A. Simmins, of the Institute of Medical Psychology (The Tavistock Clinic,) tested the patient with the Revised Stanford Binet Test and the Kohs Block Design Test. I wish to express my indebtedness to her for her invaluable aid.

I am grateful to Mr. Geoffery Jefferson for his kind co-operation and interest ; to Dr. J. G. Greenfield for the pathological material and reports ; and to Dr. E. A. Carmichael for permission to examine the patient, who was on his service, and for his most helpful guidance.

\section{REFERENCES}

Ackerly, S. (1935). Amer. J. Psychiat., 92, 717.

Brickner, R. M. (1932). Proc. Assoc. Res. nerv. ment. Dis., 13, 259.

(1936). The Intellectual Functions of the Frontal Lobes. Macmillan, New York. Burt, C. (1933). Handbook of Tests. King \& Son, London.

Cattell, R. B. (1935). Handbook for Cattell Group and Individual Intelligence Tests. Harrup and Co., London.

Downey, J. E. (1923-4). The Will Temperament and Its Testing. World Book Co., Yonkers, New York.

German, W. J., and Fox, J. C. (1932). Proc. Assoc. Res. nerv. ment. Dis., 13, 378.

Goldstein, K. (1936). J. Neurol. Psychopath., 17, 27. 
Goldstein, K., and Katz, S. E. (1937). Arch. Neurol. Psychiat., Chicago, 38, 473.

Jefferson, G. (1937). Brit. med. J., 2, 199.

Kohs, S. C. (1923). Intelligence Measurement. Macmillan, New York.

McCall, W. A., and Herring, J. P. (1937). A Comprehensive Test Program. Laidlaw Bros., Chicago.

Penfield, W., and Evans, J. P. (1932). Proc. Assoc. Res. nerv. ment. Dis., 13, 352.

- (1935). Brain, 58, 115.

Porteus, S. D. (1924). Guide to Porteus Maze Test. Publication of the Training School at Vineland, New Jersey.

Terman, L. M., and Merrill, M. A. (1937). Directions for Administering Forms L. and M. Revision of Stanford Binet Tests of Intelligence. Houghton and Mifflin, Boston.

Thurstone, L. L. (1938). Primary Mental Abilities. Psychometric Monographs No. 1. Univ. Chicago Press; Camb. Univ. Press. 